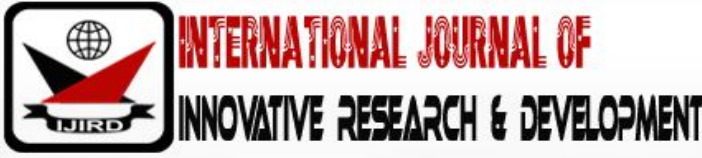

ISSN 2278 - 0211 (Online)

\section{Pragmatic Teleconsultation Network Model to Address the Bandwidth Deficiency Inhibiting Real-Time Telemedicine Implementation in the Sub-Saharan Africa}

\author{
Richard Kofi Annan \\ Assistant Lecturer, University of Mines and Technology, Tarkwa, Ghana \\ Jonas Tawiah Agyepong \\ Research and Teaching Assistant, University of Mines and Technology, Tarkwa, Ghana
}

\begin{abstract}
:
The recent Ebola outbreak in the Western Sub-Saharan Africa shows a clear case of the reliant of developing countries on foreign medicinal experts to combat unprepared diseases outbreak. This has raised much worry about the confinement of medical experts in developing nations. The seriousness of the outbreak infectiousness, frightened the western world to release their specialists readily to help in numbers; thus, the protracted mitigation quick-fix which costed the lives of numerous. Distant video auditing telemedicine initiatives over telecommunication networks would have been helpful to assessing the Ebola alleviation other than the stringent wellbeing Personal Protection Equipment (PPE) which was not that powerful. Despite the usefulness of telemedicine in the Sub-Saharan Africa, evidence to show the effectiveness of its real-time implementation is highly limited. Hence, this research focused on investigating the factors repressing its implementation in this region. Emerging from the research was the limitation of published studies to tackle the bandwidth limitation which is the key element not making the real-time dominance telemedicine practical in the Sub-Saharan Africa. To add knowledge to solving the bandwidth limitation, the project leveraged on Quality of Service technologies and Video calling techniques over IP network to present a solution, by developing a pragmatic network prototype supporting telemedicine in real-time. The designed, developed and evaluated network prototype has ascertained that, in the Sub-Saharan Africa using a case in Ghana with a bandwidth of 512kbps real-time telemedicine is feasible in High Definition video Quality.
\end{abstract}

Keywords: Telemedicine, teleconsultation, Sub-Saharan Africa, telecommunication networks, quality of service, internet protocol, low-resource settings

\section{Introduction}

In recent years, studies examining means to improve the healthcare delivery in developing countries focuses on the use of ICT initiatives as commonly known as telemedicine (for example telemedicine) to make access to health care easy and timely (Huaynate et al., 2015; Mars, 2013). Be that as it may, the instability of basic infrastructure to support the telemedicine initiatives in developing countries has not been bettered as well as the poor planning and the deficiency of ICT training. In the context of this study, the technological framework limiting telemedicine initiation in the Sub-Saharan Africa includes unstable communication network and electricity power supply. In the Sub-Saharan Africa, the unstable power supply, unstable network connectivity time coupled with the bandwidth limitation belittles the genuine potential of communication networks to support health systems e.g. teleconsultation in the context of this study. Several authors have highlighted the impact of this setback in their telemedicine initiative in the Sub-Saharan Africa briefly reported below.

\section{Literature Review}

In (Geissbuhler et al., 2003), prior telemedicine experiences reported in Senegal including the use of ISDN-based telemedicine for cardiology and neurology diagnosis between Dakar and Tambocounda regions. Similarly, the electricity power supply, unavailability of reliable connectivity coupled with bandwidth limitation beyond large city confined the effectiveness of their telemedicine implementation only to the urban communities in the country.

Furthermore, in Zambia, the remote villages are provided with telemedicine applications to reduce the morbidity and mortality rates. The rationale behind the telemedicine implementation in villages was to balance the health care access in the urban and rural areas as a result of the restriction of the effective communication network, internet connectivity and electricity supply to only the cities (Mupela, Mustarde, \& Jones, 2011). 
To portray a glimpse of telemedicine in the Sub-Saharan Africa, the "Réseau en Afrique Francophone pour la Télémédecine" (RAFT) telemedicine network is noted. The RAFT primarily operates in 13, 5, and 1, French, English, and Portuguese speaking countries, respectively (Bediang et al., 2014) for example Cameroon and Ivory Coast. Its emphasis is on webcasts interactive courses for knowledge sharing across health professionals usually in the form of presentations and dialogs between experts in different countries. Also, in RAFT countries, collaborative medical diagnosis include ultrasound, electrocardiography, and the likes of which are all asynchronous (not real-time). To make better RAFT operations in operating country, the projection of $3 \mathrm{G}$ internet connection is mentioned to connect site together which arguably may support the synchronous (real-time) telemedicine.

The onset realization of Telemedicine in Sub-Saharan Africa will not be dominant if bandwidth, network coverage, and power supply are not guaranteed. To find solutions to the long lasting basic infrastructure problem, Van-Gurp et al. (2015) claimed that telemedicine can only be useful in Africa if low tech solutions that work are applied to the bandwidth limitation problems by focusing on non-synchronous online communication. For example, sending medical information via emails online does not consume much bandwidth compared to sending similar information using online video calling application. However, this study challenges the claim of Van-Gurp et al. (2015) that asynchronous telemedicine is not the ideal solution for the SubSaharan Africa region despite the bandwidth limitation. Synchronous telemedicine is relevant in the Sub-Saharan Africa provided the right techniques to support its quality delivery over telecommunication network be identifiable and pragmatically applicable in deficient bandwidth settings.

From the above report, it is evident that the Sub-Saharan Africa needs telemedicine (Mars, 2013), and prior initiations have been taking to make it effective. Many telemedicine applications in this region are propelled by the need for collaborative medical diagnosis remotely. As the result of the bandwidth limitation in the Sub-Saharan Africa as established earlier, many countries (in the previous analysis) finds the asynchronous telemedicine as useful for medical diagnosis collaborations as claimed by Van-Gurp et al. (2015). Thus, the insufficient bandwidth in this region limits synchronous telemedicine considering the need to overcome delivery delay, jitter, and data loss over IP telecommunication networks. To argue this claim, another group of study is of the standpoints that, increasing bandwidth does not necessarily guarantee minimized end-to-end delay and information loss over IP-based networks (Chimmanee, 2013; "Cisco", 2008). Thereby, implying the significance of further research to making synchronous telemedicine realism is the Sub-Saharan African Region despite the bandwidth challenge.

\subsection{Synchronous Teleconsulation Network Model}

In order to empirically design and develop the synchronous teleconsultation network model as anticipated for remote medicinal usage in the Sub-Saharan Africa for good, the following technologies were recognized as useful to overcome the bandwidth limitation problem in region; DiffServ QoS, IP multimedia protocols (Real-time Transport Protocol (RTP), Session Initiation Protocol (SIP) and video compression standards.

According to Da-Silva (2012), Real-time video call for remote teleconsultation is bandwidth intensive compared to sharing clinical information during a collaborative consultation session. Therefore, synchronous teleconsultation is not feasible on IP best effort networks (Xiao-dan et al., 2012). To provide such preferential treat for teleconsultation video applications and its associated audio the DiffServ QoS mechanisms in (Szigeti, 2013; Black \& Jones, 2015) are needful. Thus, identifying traffic types on the network, aggregating them into classes by marking and applying per-hop-behavior (preferential bandwidth allocation treatment for traffic classes) mechanisms such as scheduling, shaping and policing. The DiffServ QoS is used in the event of network congestion where multimedia traffic (audio, video, data) compete for transmission over limited bandwidth links (e.g. the WAN segment). Without, the DiffServ QoS application all traffic is treated equally using the IP best effort forwarding. A typical teleconsultation multimedia (video, data, audio) session is life and time critical and requires special transmission treatment (Kalam, Ferreira, \& Kratz, 2016). Therefore, to achieve delivery quality for the teleconsultation application in this research DiffServ QoS mechanisms are crucial (Da-Silva, 2012).

The RTP protocol (Lusilao-Zodi et al., 2011) is the packet format for the interactive teleconsultation multimedia traffic streams providing end-to-end transport services having real-time features. A typical RTP stream comprises of the control (RTCP) and data (a transfer protocol supporting real-time features such as synchronization, detecting packet loss, security, payload identification) (Liang \& Shun, 2010).

Taking into consideration the quick outlook change from the ordinary telephony (thus circuit switched telephony) to packet switching advancements (IP-based network services), network-centered multimedia communication applications have created an enormous effect on our regular activities. By way of illustration, Hans et al., (2016) show how its application types as in IP-telephony (VoIP), streaming, IP-television, and medical services such as real-time telemedicine application (e.g. telesurgery and tele-consultation) improve life experiences. The signaling protocol considered in this publication is SIP to implement real-time teleconsultation session over IP the anticipated teleconsultation network model design (Rattal, Badri, \& Moughit, 2013). SIP is defined in the RFC (RFCs 326, 2543, 3261) as an application layer protocol by the IETF (Da-Silva, 2012). The consideration of SIP in for the teleconsultation network model design as also in (Barz \& Bassett, 2016) pivots on lone regular session setup and nothing else and also the capability of its deployment to initiating additional non-telephony sessions such as interactive video calling (e.g. interactive teleconsultation via video calling). Another consideration of SIP is its effectiveness for the deployment for multimedia transmission over IP-based networks.

The next section would incorporate the mentioned technologies so as to design and develop a network model as a prototype to support real-time teleconsultation via video calls under bandwidth constrained conditions. 


\section{Teleconsultation Network Model Design}

The basis of Internet Protocol (IP) network connectivity options depend on the objective of accomplishment; thus, the teleconsultation in low-resource setting taking into consideration quality, teleconsultation location and cost (Hadeed, Holcomb, \& Latifi, 2010). During a clinical teleconsultation session, a considerable amount of bandwidth needs to be guaranteed depending on the video and image per session at the expense of another clinical service to attain an appreciable quality (Da-Silva, 2012). In the instance where connecting two hospital sites, a land-based (leased lines e.g. T1 or T3) connectivity solution applicability would be worthy although up service and speed is guaranteed, it is costly (Hadeed, Holcomb, \& Latifi, 2010). However, in the rural areas where the land-based solution would be difficult to obtain, a quick connection via local Internet Service Provider (ISP) will be the best option since they moderate blend of cost and speed.

The network model design recommended (Figure 1) would have the blend of hospital-to-hospital broadband connection (e.g. 3G mobile broadband enabled devices or DSL) via ISP WAN with VPN enabled for security and a dedicated leased line (T1 or T3) WAN connection. The aggregate connectivity option grants high availability and redundancy option. The broadband connection could be used for data services while dedicating the leased-line option for teleconsultation via video calling. The leased line provides guaranteed high-speed, reliable, and configuration flexibility. The limitation to this recommendation would be cost most especially, the leased line option. However, the design strategy is to identify what minimum bandwidth can support the two High Definition (HD) teleconsultation video call sessions as a logical means to cut down medical cost. Besides, telemedicine is life and time critical (Cadger et al., 2016); therefore, health bodies seeking to benefit from its applications should be able to bear the cost of implementing it the right way in order to save lives rather than losing them as a result of poor quality delivery.

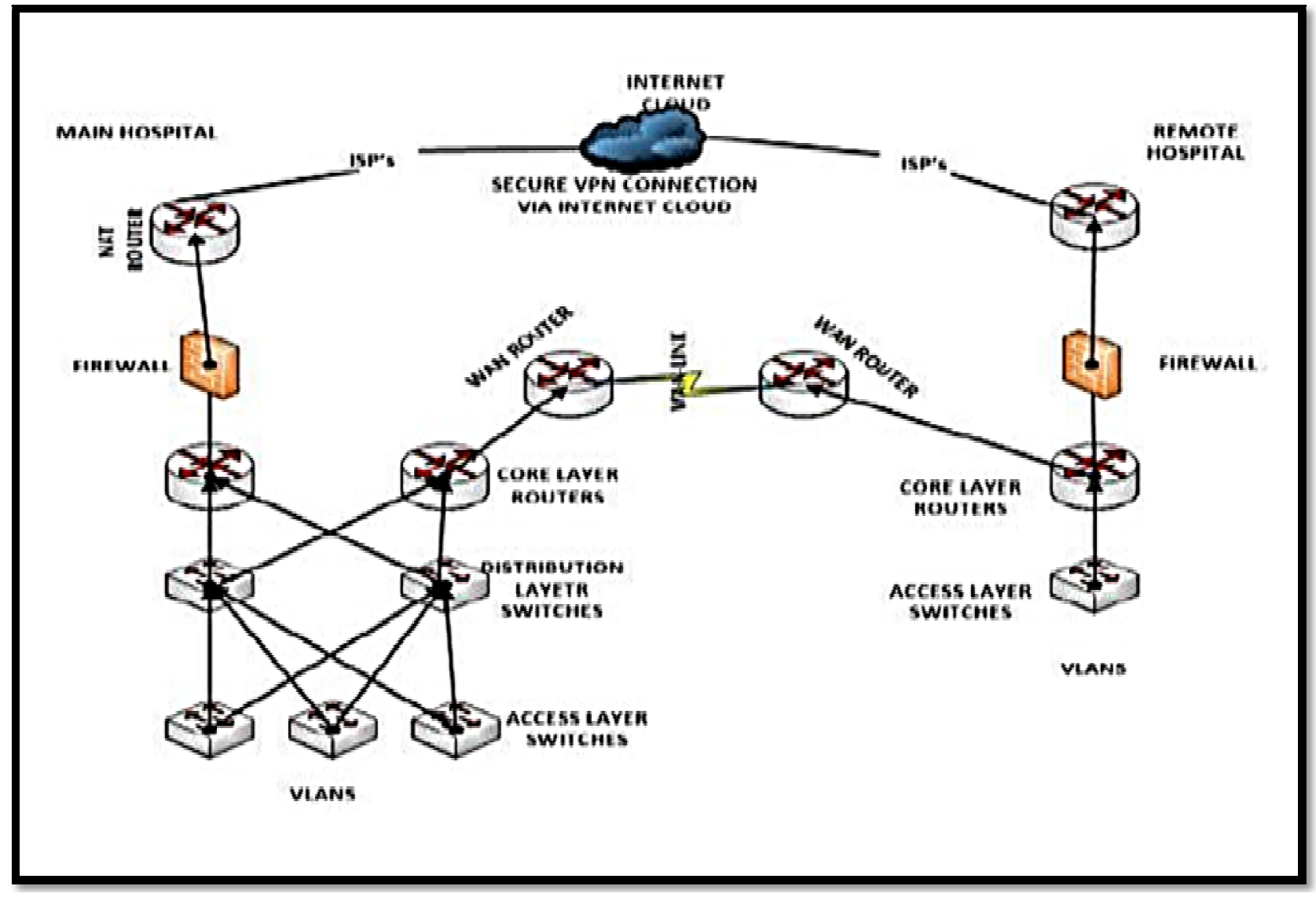

Figure 1: Recommended Teleconsultation Network Model Diagram

The network prototype is condensed as follows focusing more on the main connectivity option (thus the dedicated leased-line option) as considered ideal for hospital-to- hospital teleconsultation (Hadeed, Holcomb, \& Latifi, 2010) as recommended earlier. The design is shown below as in Figure 3. 


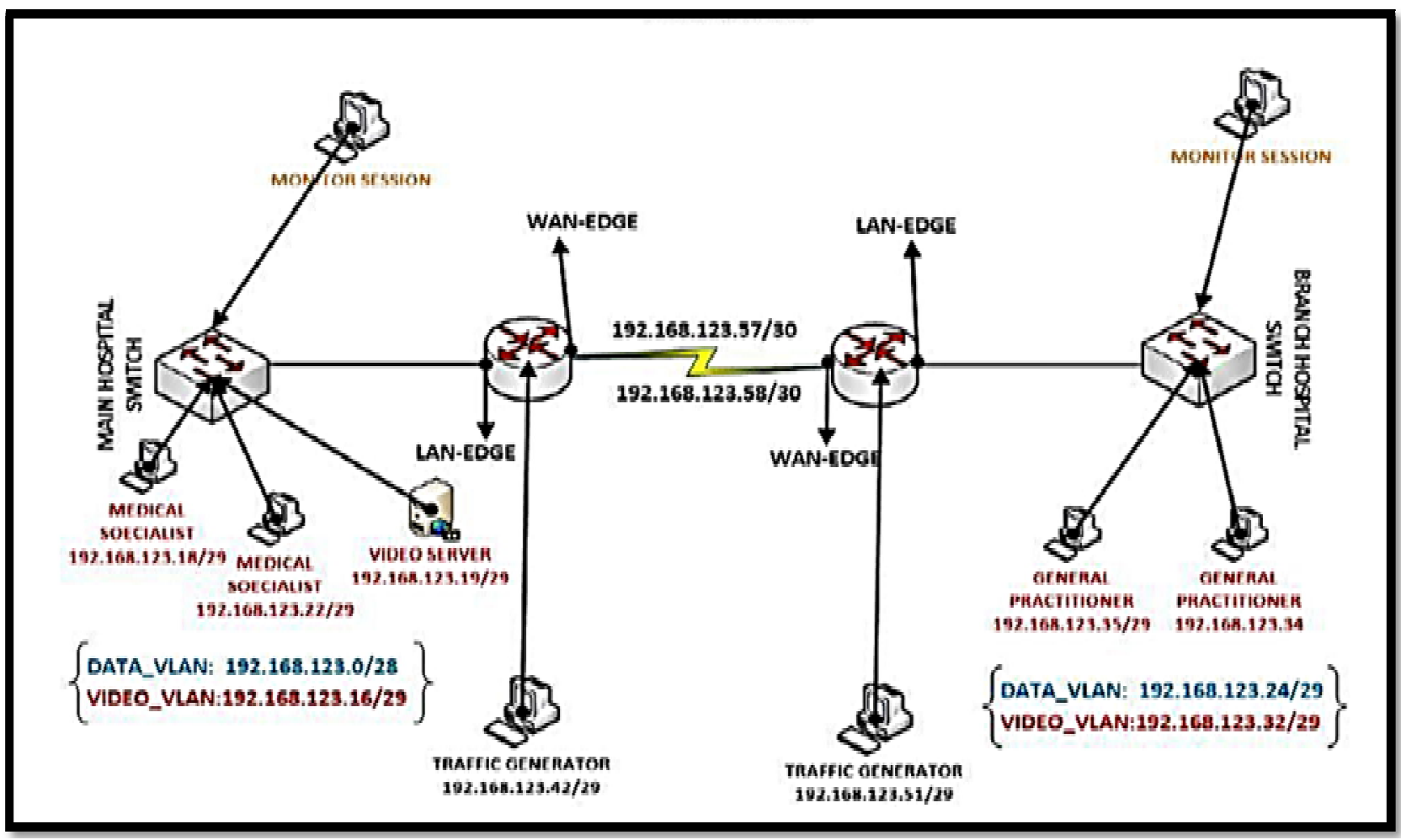

Figure 2: Teleconsultation Network Model Topology

To envision the teleconsultation via video calling from the networking perspective, the network domains providing medicinal specialists services and only General Practitioner (GP) services represents the main hospital and remote hospital respectively. Each network domain has core router aggregating LAN traffic traversing over the traditional WAN segment (e.g. dedicated leased-line) to the other (refer to Figure 1). Furthermore, the traffic generator station serves two purposes in this network model; thus, generating traffic across the WAN link to each site and monitoring bandwidth threshold. The two data traffic considered in this study includes teleconsultation data traffic and clinical transaction traffic. For example, traffic between the two-traffic generator end-stations represents the clinical data and traffic generated to the Video VLAN (teleconsultation subnet) signifies the teleconsultation data traffic. Also, On the edge-router in each network domain, Simple Network Management Protocol (SNMP) server is set up to collect network information (bandwidth utilization) from network end-stations; and also identifies which protocol are consuming the most bandwidth on the WAN-link. The SNMP server exports the information obtained to the traffic generator end-station hosting the net-flow monitoring software (Solar-Winds real-time net-flow analyser) in each domain.

\subsection{Teleconsultation Video Calling Set-up}

To implementing video calling for teleconsultation, the 3Cx VoIP server-client application was used. The server acts as the call admission control in the context of this study. Only registered health professionals are allowed for video calling session (thus, remote GPs and the two main- hospital medical specialists.

\subsection{The QOS Classification and Marking Implementation}

In a typical Hospital environment, the real-time video call teleconsultation supported network would include video, voice, and clinical data. Video, voice and data traffic during teleconsultation session is critical compared to clinical data exchange traffic (Kalam, Ferreira, \& Kratz, 2016). Also, while teleconsultation session proceeds video and voice have priority over data traffic in the same session for example doctors exchanging data while having video calls. However, the interactive video and voice of the teleconsultation session are giving similar priority for synchronization reasons (Black \& Jones, 2015). Thus, voice and video should be received simultaneously for rendering specific medical instructions.

The classification of the synchronous teleconsultation traffic, as well as marking, is done as close to the network edge. For each site (refer to Figure 2) ingress and egress classification and marking are done at the LAN edge and WAN edge respectively. The DSCP value markings are used to classify and condition (Black \& Jones, 2015) and to outline the per-hop behaviour of each multimedia traffic classification. Table 1 shows the traffic classification and marking for the teleconsultation network model design. Teleconsultation interactive video session is marked EF, teleconsultation data AF41, clinical data AF31 and network management AF32. The teleconsultation video call session has the highest priority as established synchronous teleconsultation are delay-sensitive (Szigeti, 2013). Also, teleconsultation data is given priority over the normal clinical data 
transactions; because, the asynchronous teleconsultation is life critical and hence considered as mission critical data in this network model.

\begin{tabular}{|c|c|}
\hline Network Traffic & AF DSCP Coding \\
\hline $\begin{array}{c}\text { Teleconsultation video and } \\
\text { audio traffic }\end{array}$ & EF \\
\hline Teleconsultation data traffic & AF41 \\
\hline Internal clinical data traffic & AF31 \\
\hline Network management (e.g. Ping) & AF32 \\
\hline
\end{tabular}

Table 1: Teleconsultation Network Model Traffic

Classification and Marking

The anticipated bandwidth distribution allocation for the traffic classification and marking is shown in Figure 4 below;

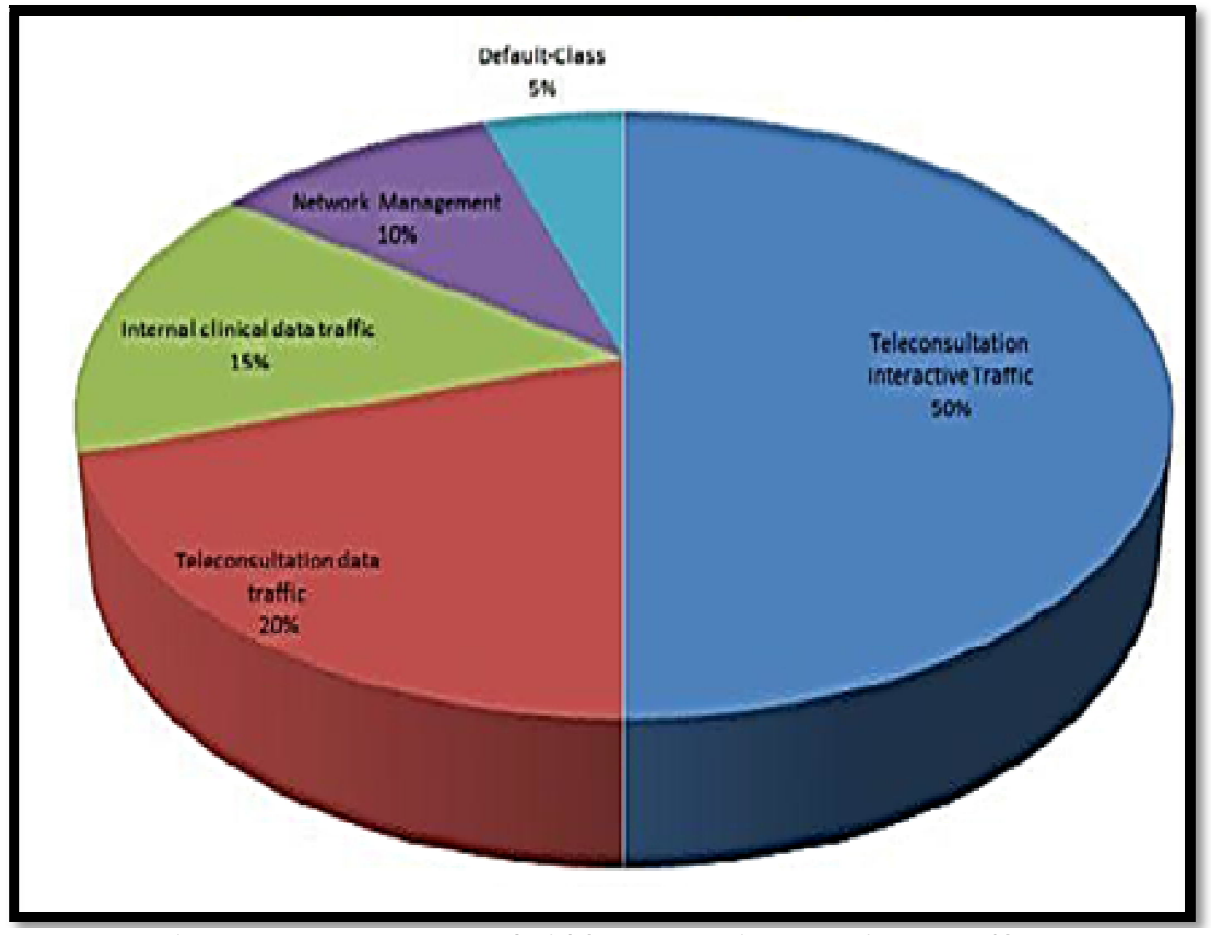

Figure 3: Transport Bandwidth Reservation Requirement Chart

\section{Discussion of the Teleconsultation Network Model Test Result}

The test parameter was set up to demonstrate two teleconsultation practices with one showing an X-ray still image and the other fast motion (patient rubbing skin as an example of dermatological diagnosis) video calling. All the video was transmitted in High Definition requiring transport bandwidth of 1024kbps in both end station. The Research established that first-time teleconsultation user experience is crucial for its acceptance and continuous utilization (Chopra, 2015) as similar to the hospital clients' high-quality teleconsultation video requirement.

The result of the first test run indicated that synchronous teleconsultation is bandwidth intensive as was established in (Hadeed, Holcomb, \& Latifi, 2010) especially when transmitting in high video resolutions. Under no network congestion conditions, both sessions had excellent perceived user video quality. However, the $\mathrm{HD}$ fast motion teleconsultation video session test consumed much bandwidth compared to the still image session. The assumption was that under high network congestion conditions, the fast motion teleconsultation would be much affected compared to the still image regarding perceived user video quality. The assumption was evident in the third test with the QoS policy reserving 50\% bandwidth for teleconsultation video sessions. The perceived user video quality based on International Telecommunication Union Mean Score Opinion (MSO) for interoperating video quality, for the regular motion teleconsultation video session was fair with slightly "annoying" video impairment compared to the still image video session with good and perceptible but not "annoying" perceived user video quality. Again, with this same QoS policy the regular motion video end stations consumed 3\% more bandwidth capacity than the still image utilizing $29 \%$ bandwidth However, when the regular motion session was run individually under same network congestion setting, the perceived user video quality was good with perceptible impairment but not "annoying." 
The results obtained indicated that the QoS policy reserving $50 \%$ does not meet up with the two concurrent teleconsultation video call session requirements. Therefore, there was the need for augmenting the bandwidth reservation for teleconsultation video session. However, the inference from this QoS policy is that on a 1024kbps link reserving priority bandwidth of $512 \mathrm{kbps}$ can only support one $\mathrm{HD}$ teleconsultation video session be it still image of regular motion video session. The priority bandwidth reservation for teleconsultation was regulated steadily to $80 \%$. At this point, real user perceived video quality with perceptible but not "annoying" and imperceptible impairments was obtained for the regular motion and still image teleconsultation video sessions respectively running them simultaneously. Although this QoS policy augmentation meets the client's requirement it does so the expense of starving all the other TCP data traffic transaction.

The test concluded on the note that with $1024 \mathrm{kbps}$ bandwidth link reserving 50\% for synchronous teleconsultation traffic cannot run two concurrent HD video according to this test results obtained. The reason is that synchronous video calling with significant and regular packet loss affect video call session experience (Szigeti, 2013). Also, in agreement with Kalam, Ferreira, and Kratz, (2016) teleconsultation is life and time critical. As a result, the network developments considered fit for its application should be reliable and anti-error-prone. The augmented $80 \%$ bandwidth reservation for teleconsultation video session works perfect but starves other data transactions. To overcome the limitation, the clinics can resort to the connectivity recommendation in the design section as also recommended by Hadeed, Holcomb and Latifi (2010) which is the discovered future work that needs further research and test evaluation.

\section{Conclusions and Recommendation}

In view of this now study, it is promising to state that synchronous telemedicine is achievable in the low-resource settings in the Sub-Saharan Africa as good. The research followed the argument that increasing bandwidth does not necessarily guarantee real-time telemedicine service delivery quality over IP networks. The evidence from this study suggest that incorporating Quality of Service and real-time IP-based multimedia technologies on telecommunication network systems supporting medical services, synchronous telemedicine is plausible bandwidth deficient areas. Furthermore, the present study has gone some way towards enhancing the knowledge of addressing the technological factor i.e. bandwidth constraints repressing the dominance of real-time telemedicine application in the Sub-Saharan Africa region. Also, the findings from the research complement those earlier studies, raising concerns about the limitation of health professionals in developing countries by presenting them with a pragmatic solution to supplement the medical specialist work force with teleconsultation. For example, with this research's teleconsultation network model, clinics in the Sub-Saharan Africa region considering to takeup teleconsultation initiative can adhere to this model to run simultaneous specialist services between two or more remote sites.

The result of this study indicates that, under bandwidth constraints, with QoS the 1024kbps WAN-link connecting two hospital sites can support two concurrent HD synchronous teleconsultation video calling sessions and one on 512kbps link capacity be it still image or regular motion teleconsultation video. It is unfortunate that the study did not include the video compression codecs as anticipated as a result of the lack of funds to obtaining licensed video calling applications with such features; and also, the video quality measurement was based on generalized objective user perceptions.

More research using the controlled subjective video quality assessment trials that covers range of video resolution bandwidth and the recent H.265 and VP9 video compression codec can further allow the 512kbps to support two simultaneous teleconsultation video call sessions or even more. Also, another reasonable approach to tackle this issue besides video compression is to supplement the 512kbps link with wireless broadband technology such $3 \mathrm{G}$ and $4 \mathrm{G}$ and load-balance clinical and teleconsultation video traffic on the aggregated links.

\section{References}

i. Barz, H., \& Bassett, G. (2016). Multimedia networks. Chicester: John Wiley and Sons Ltd., 418.

ii. Bediang, G., Perrin, C., Ruiz de Castañeda, R., Kamga, Y., Sawadogo, A., Bagayoko, C.,...Geissbuhler, A. (2014). The RAFT telemedicine network: lessons learnt and perspectives from a decade of educational and clinical services in low- and middle-incomes countries. Frontiers in Public Health, 2, 1-6.

iii. Black, D., \& Jones, P. (2015). Differentiated services (diffserv) and real-time communication. Informational-Internet Engineering Task Force, 1-26.

iv. Cadger, F., Curran, K., Santos, J., \& Moffett, S. (2016). Location and mobility-aware routing for multimedia streaming in disaster telemedicine. Ad Hoc Networks, 36, 332-348.

v. Chimmanee, S. (2013). PACS metric based on regression for evaluating end-to-end QoS capability over the internet for telemedicine. International Conference on Information Networking, 359-364.

vi. Cisco: Implementing quality of service policies with DSCP. (2008). Retrieved 2016, May 3 from http:/ / www.cisco.com/ c/ en/ us/ support/ docs/ quality-of-service-qos/ qos-packet-

marking/ 10103dscpvalues.html?referring_site=RE\&pos=1\&page=http:// www.cisco.com/ c/ en/ us/ td/ docs/ ios/12_2 / qos/ configuration/ guide/ fqos_c/ qcfdfsrv.html

vii. Chopra, A. (2015). Quality of service-concept and need for wireless telemedicine and e-health services. International Journal of Computer Networks and Communications Security, 3(5), 205-207.

viii. Da-Silva, M. (2012). Multimedia communications and networking. Boca Raton, FL: CRC Press, 506. 
ix. Geissbuhler, A., et al. (2003). Telemedicine in Western Africa: lessons learned from a pilot project in Mali, perspectives and recommendations. In AMIA 2003 Synposium Proceedings, Geneva, 249-253.

x. Huaynate, A.C., Pajuelo, T.M., Correa, M., Mayta M.H., Oberhelman, R., Murphy, L., Paz-Soldan, V. (2015). Diagnostics barriers and innovations in rural areas: insights from junior medical doctors on the frontlines of rural care in Peru. BMC Health Services Research, 1(15), 1-10.

xi. Hadeed, G., Holcomb, M., \& Latifi, R. (2010). Communication technologies: An overview of telemedicine connectivity. In Latifi, R. (Ed.), Telemedicine for Trauma, Emergencies, and Disaster Management, 1st Ed, (pp. 37-50). Boston: Artech House.

xii. Kalam, A.E., Ferreira, A., \& Kratz, F. (2016). Bilateral teleoperation system using QoS and secure communication networks for telemedicine applications. IEEE Systems Journal, 10(2), 709-720.

xiii. Liang, P., \& Shun, Y. (2010). Research and implementation of voice transmission based on RTP protocol. In Computational Problem-Solving (ICCP), 2010 International Conference. Lijiang: IEEE, 416-419.

xiv. Lusilao-Zodi, G., et al., (2011). RRB-SIMD: RTP rate-based SIMD protocol for media streaming applications over the internet. In 2011 Ninth Annual Communication Networks and Services Research Conference. Ottawa, 69-76.

xv. Mars, M. (2013). Telemedicine and advances in urban and rural healthcare delivery in Africa, progress in cardiovascular diseases. ScienceDirect, 56(3), 326-335.

xvi. Mupela, E.N., Mustarde, P., \& Jones, H.L. (2011). Telemedicine in primary health: The Virtual Doctor Project Zambia. Philosophy, Ethics, and Humanities in Medicine 2011, 6(9), 1-8.

xvii. Rattal, S., Badri, A., \& Moughit, M. (2013). Performance analysis of hybrid codecs G. 711 and G. 729 over signaling protocols H. 323 and SIP. International Journal of Computer Applications, 72(3), 30-33.

xviii. Szigeti, T. (2013). End-to-end QoS network design. Indianapolis, IN: Cisco Press, 1024.

xix. Van-Gurp, J., Soyannwo, O., Odebunmi, K., Dania, S., van Selm, M., van Leeuwen, E., Vissers, K.,...Hasselaar, J. (2015). Telemedicine's potential to support good dying in Nigeria: A qualitative study. PLOS ONE, 10(6), 1-15.

xx. Xiao-dan, D., Qing, H., Yong-hong, L., \& Hong, Y. (2012). The system construction and the implementation of QOS control mechanism in intelligent streaming media. Physics Procedia, 25, 808-813. 